\title{
Propriétés physico-chimiques, fonctionnelles et microbiologiques de la farine de maïs germé enrichie de larves d'insectes comestibles Rhynchophorus phoenicis et Oryctes owariensis
}

\author{
Angaman Djédoux Maxime ${ }^{1 *}$, Ehouman Ano Guy serge ${ }^{1}$, Boko Adjoua Christiane Eunice ${ }^{1}$ \\ ${ }^{1}$ Laboratoire d'Agrovalorisation, Département Biochimie-Microbiologie, UFR Agroforesterie, Université Jean \\ Lorougnon Guédé de Daloa, BP 150 Daloa, Côte d'Ivoire. \\ Auteur correspondant, e-mail : angaman@ujlg.edu.ci
}

Original submitted in on $18^{\text {th }}$ December 2020. Published online at www.m.elewa.org/journals/ on $28^{\text {th }}$ February 2021 https://doi.org/10.35759/JABs.158.5

\begin{abstract}
RESUME
Objectif : L'objectif de cette étude est d'évaluer les propriétés de quelques farines formulées à partir de maïs germé enrichi de larves de Rhynchophorus phoenicis (charançon africain du palmier) et de Oryctes owariencis (scarabée rhinocéros).

Méthodologie et Résultats : Pour ce faire, différentes compositions de farines de maïs germé et de poudre de larves (PL) ont été préparées comme suit : les farines composées lot1 (maïs 80\% + PL20\%), lot2 (maïs $78,5 \%+$ PL22,5\%), lot3 (maïs 75\% + PL25\%). Les caractéristiques physico-chimiques, les propriétés fonctionnelles et microbiologiques de ces farines ont été déterminées selon les méthodes standards. Les résultats ont révélé que l'incorporation de la poudre de larves comestibles à la farine de maïs a amélioré significativement les paramètres biochimiques notamment la teneur en protéines, en matière grasse et la valeur énergétique. Ainsi, les farines de maïs germé enrichies avec Oryctes owariensis ont présenté une teneur plus élevée en protéines allant jusqu'à $29,73 \pm 5,87 \%$ pour le FMaGO25. Cependant, celles enrichies au Rhynchophorus phoenicis avaient des taux de matières grasses plus élevées estimées à $21,24 \pm 0,772 \%$ avec 466,67 $\pm 9,325 \mathrm{kcal} / 100 \mathrm{MS}$ pour la FMaGR 22,5. Toutefois, seule la farine FMaGR20 est proche du standard établi par le codex alimentarius. Aussi, Les charges microbiologiques détectées dans ces farines ont été inférieures aux normes microbiologiques applicables aux farines.

Conclusion et application : L'incorporation des larves de Oryctes owariensis et de Rhynchophorus phoenicis a permis d'obtenir une farine de maïs enrichi. Ces formulations pourront donc alors être recommandées dans la lutte contre les carences nutritionnelles, Ainsi la FMaGR20 contribuera à lutter contre la malnutrition infantile.
\end{abstract}

Motsclés : Farine améliorée, maïs germé, larves, Rhynchophorus phoenicis, Oryctes owariensis, d'insectes comestibles 
Angaman et al., J. Appl. Biosci. 2021 Propriétés physico-chimiques, fonctionnelles et microbiologiques de la farine de maïs germé enrichie de larves d'insectes comestibles Rhynchophorus phoenicis et Oryctes owariensis

\title{
Physicochemical, functional and microbiological properties of sprouted corn flour enriched with larvae of Edible insects Rhynchophorus phoenicis and Oryctes owariensis
}

\begin{abstract}
Objective: The objective of this study is to evaluate the properties of the flour formulated from enriched sprouted corn, Rhynchophorus phoenicis and larvae of Oryctes owariencis.

Methodology and Results: To do this, different compositions of sprouted corn flours and larval powder (PL) were prepared as follows: the compound flours lot1 (corn $80 \%+$ PL20\%), lot2 (corn $78.5 \%+$ PL22.5\%), lot3 (corn 75\% + PL25\%) The physicochemical characteristics, functional and microbiological properties of these flours were determined according to standard methods. The results revealed that the incorporation of the powder of edible larvae in corn flour significantly improved the biochemical parameters including the protein content, fat content and energy value. Thus, sprouted corn flours enriched with Oryctes owariensis exhibited a higher protein content of up to $29.73 \pm 5.87 \%$ for FMaGO25. However, those enriched with Rhynchophorus phoenicis had higher fat levels estimated at $21.24 \pm 0.772 \%$ with $466.67 \pm 9.325 \mathrm{kcal} / 100 \mathrm{MS}$ for FMaGR 22.5. However, only FMaGR20 flour is close to the standard established by Codex Alimentarius. Microbiological loads detected in these flours were lower than standards applicable to flours.

Conclusion and application: Incorporation of the larvae of Oryctes owariensis and Rhynchophorus phoenicis made it possible to obtain an enriched corn flour. These formulations can therefore be recommended in the fight against nutritional deficiencies. Thus, FMaGR20 will contribute to the fight against child malnutrition.

Keywords: Improved flour, sprouted corn, larvae, Rhynchophorus phoenicis, Oryctes owariensis, Edible insects
\end{abstract}

\section{INTRODUCTION}

Malgré les efforts fournis par les Nations Unies pour l'éradication de la faim et la malnutrition dans le monde, l'insécurité alimentaire continue à gagner du terrain ces dernières décennies. Le rapport sur l'état de la sécurité alimentaire et de la nutrition dans le monde publié conjointement par la FAO, la FIDA, I'UNICEF, le PAM et l'OMS en 2019, chiffre à plus de 820 millions le nombre de personnes en situation d'insécurité alimentaire dans le monde (FAO, 2019). La forme la plus grave de ces problèmes nutritionnels est la malnutrition protéinoénergétique qui touche présentement plus d'un milliard de personnes (FAO, 2019). Elle est actuellement très présente dans les pays pauvres notamment sur le continent africain où elle touche environ une personne sur cinq. En Côte d'Ivoire par exemple, les résultats de l'enquête SMART 2011. 2012 révèlent $29,8 \%$ des enfants souffrant de malnutrition chronique et $7,5 \%$ d'enfant atteint d'émaciation (EDS-MICS, 2012). La cause principale de cette malnutrition serait liée à un déséquilibre de l'apport énergétique, protéique et/ou nutritif. En effet, le régime alimentaire est peu diversifié, essentiellement basé sur les tubercules, les racines et les céréales qui contribuent à plus de $65 \%$ aux apports énergétiques alimentaires journaliers (INS et ICF, 2012). Cependant, ces aliments sont généralement pauvres en certains nutriments tels que les protéines, les lipides, les minéraux et vitamines dont les apports sont indispensables pour assurer l'équilibre nutritionnel de l'organisme. Une des voies les plus viables pour couvrir ces besoins nutritionnels est l'enrichissement de ces aliments énergétiques par des aliments de complément riches en protéines, lipides, vitamines et minéraux. Parmi ces aliments de compléments, les insectes comestibles recommandés par la FAO pour nourrir le monde d'ici à 2050 s'avèrent être une alternative convenable. De nombreux travaux ont montré que les insectes comestibles très riches en éléments nutritifs notamment en protéines, lipides, vitamines et minéraux peuvent aider à pallier certaines carences en nutriments. En Côte d'Ivoire, neuf espèces d'insectes sont consommées par une frange de la population en remplacement de la viande et du poisson (Ehounou et al., 2018). Parmi celles-ci, d'autres sont beaucoup appréciées sous 
leur stade larvaire pour leur goût et leur arôme c'est le cas des espèces Rynchophorus phoenicis, et Oryctes owariensis (Le Gall, 2015). Au-delà de ces qualités organoleptiques, ces larves renferment de nombreux atouts sur le plan nutritionnel et rhéologique pouvant permettre de formuler des

\section{MATERIEL ET METHODES}

Le matériel biologique est constitué des larves de Rhynchophorus phoenicis et de Oryctes owariensis collectées respectivement dans les palmiers et raphias de la ville Daloa. Ainsi que de grains de maïs achetés directement au grand marché de ladite ville.

Production des farines de Maïs et Mil germés : Les grains de Maïs ont été lavés puis trempés séparément dans un récipient ouvert pendant $48 \mathrm{~h}$ puis étalés sur un tissu en coton humidifié pendant 3 jours pour la germination. Ensuite les grains qui ont effectivement germés ont été séchés à $55^{\circ} \mathrm{C}$ à l'étuve pendant $72 \mathrm{~h}$ puis débarrassés de leurs plantules avant d'être finement broyés avec un broyeur de type Forplex et tamisés avec un tamis de diamètre $500 \mu \mathrm{m}$. La farine de Maïs germé obtenu a été conditionné dans un Bole en plastique codée FMaG. aliments stables et riches en nutriments. C'est dans ce cadre qu'interviendra notre étude, sur l'élaboration de farines enrichies aux poudres de larves de Rhynchophorus phoenicis et de Oryctes owariensis afin d'en évaluer les potentialités nutritionnelles et la qualité microbiologique.

Production des poudres de Rhynchophorus phoenicis et Oryctes owariensis : Après la collecte, les larves ont été conservées séparément à $-80^{\circ} \mathrm{C}$. Les larves décongelées ont été nettoyées à l'eau distillée, égouttées et séchées à $50^{\circ} \mathrm{C}$ dans un four pendant 72 h. Les larves $(300 \mathrm{~g})$ séchées ont été broyées à l'aide d'un broyeur pour obtenir la farine brute conditionnée et conservée.

Élaboration de farines incorporées de poudre d'insectes : Les farines enrichies sont obtenues en incorporant dans la farine de maïs à différentes proportions de poudre de 0 . owariensis ou de $R$. phoenicis (tableau I). Les lots (1, 2 et 3 ) enrichis au $R$. phoenicis sont respectivement codés FMaGR20; FMaGR22.5 et FMaGR25. Par contre, ceux enrichis avec 0 . owariensis sont codés FMaGO20 ; FMaGO22, 5 et $\mathrm{FMaGO} 25$.

Tableau 1 : Différentes proportions pour $100 \mathrm{~g}$ de farines enrichies

\begin{tabular}{l|l|l|l}
\hline Lot & 1 & 2 & 3 \\
\hline Farine de céréale & 80 & 77,5 & 75 \\
Poudre d'insecte & 20 & 22,5 & 25 \\
\hline
\end{tabular}

Méthodes d'analyses physico-chimiques : Le pH et l'humidité ont été déterminés selon la méthode AOAC (2000) et les cendres selon la méthode NF V03-760 (1982). L'acidité titrable a été déterminée par titrage avec une solution d'hydroxyde de sodium $(0,1 \mathrm{~N})$ en présence de phénolphtaléine (NFV05-101, 1974). La matière sèche (MS) a été obtenue par séchage à l'étuve selon la méthode décrite par l'AOAC (1990). Le degré Brix a été identifié par un réfractomètre selon la méthode de Monrose (2009). La teneur en lipides a été estimée par la méthode Soxhlet (AACC, 1984). La teneur en vitamine $C$ des extraits d'insectes a été déterminée selon la méthode décrite par Elgamouz (2016). La méthode de Kjeldahl (BIPEA.1976) a été utilisée pour déterminer les protéines brutes à partir de la teneur en azote. La détermination a été effectuée selon la formule de Koné et al. (2019). La valeur énergétique correspondant à l'énergie disponible est calculée à l'aide des coefficients spécifiques d'Atwater et Benedict (1902) pour les protéines, les lipides et les glucides.

Études de quelques propriétés fonctionnelles des farines composées: L'absorption d'eau (CAE) des farines a été mesurée par la méthode de centrifugation de Sosulski (1962). Pour la détermination de l'absorption d'huile, l'activité émulsifiante (AE) et la stabilité des émulsions (SE) nous avons utilisé la méthode de Lin et al. (1974).

Analyses microbiologiques: Les germes aérobies mésophiles (GAM) ont été dénombrés selon la norme ISO 4833 : 2003. Le dénombrement des levures et des moisissures a été fait selon la norme NF V08-059 : 2002. Les anaérobies sulfito-réducteurs ont été dénombrés selon la norme ISO 7937 : 2004. Le dénombrement des coliformes totaux a été fait selon la norme ISO 4832 : 2006 et les coliformes thermo-tolérants, selon la norme NF V08-060 : 2009. 
Angaman et al., J. Appl. Biosci. 2021 Propriétés physico-chimiques, fonctionnelles et microbiologiques de la farine de maïs germé enrichie de larves d'insectes comestibles Rhynchophorus phoenicis et Oryctes owariensis

Analyses statistiques : Les données de l'étude ont été recueillies sur le logiciel Excel. Le logiciel STATISTICA 7.1 a permis de déterminer les moyennes et écart-types. Ces moyennes obtenues, ont été sujet à une analyse de

\section{RESULTATS}

Potentiel nutritionnel des farines de Maïs germé complémentées de Rhynchophorus phoenicis et Oryctes owariensis : La composition physico-chimique des farines élaborées a été déterminée et consignée dans le tableau 2. A l'analyse, les farines présentent toutes des $\mathrm{pH}$ acides. L'incorporation de la poudre de Oryctes owariensis dans le maïs germé favorise une augmentation significative du $\mathrm{pH}$ allant de 4,92 0,028 à $6,58 \pm 0,021$ pour la FMaGO22,5. De plus, les teneurs en humidité sont relativement faibles avec des valeurs variant de $5,32 \pm 2,432$ à $9,69 \pm 3,135 \%$. Des taux de matières sèches significativement élevées sont observées pour les farines FMaGR22,5 $(94,00 \pm 2,645)$; FMaGO20 $\quad(94,68 \pm 2,432)$ et $\quad F M a G 022,5$ $(93,62 \pm 1,841)$. Contrairement aux farines FMaGR22,5 et $F M a G O 22,5$, les teneurs en cendre des autres farines élaborées augmentent significativement et passent de 1,40 à $3,55 \%$. En ce qui concerne les composés nutritifs, une diminution de la teneur en glucides est observée avec l'augmentation du taux d'incorporation des farines d'insectes. Cette teneur passe de $76,74 \pm 1,445 \%$ à $55,79 \pm 2,742 \%$ pour le FMaGR25 et à $42,69 \pm 10,677 \%$ pour le FMaGO25. Toutefois l'effet inverse est observé pour les teneurs en lipides; en protéines et en valeurs énergétiques qui quant à elles, augmentent significativement. Aussi, les teneurs plus élevées en lipides sont-elles présentées par les farines FMaGR22,5 $(21,24 \pm 0,772 \%) \quad$ et FMaGR25 $(19,20 \pm 0,361 \%)$ avec respectivement des valeurs énergétiques de $466,67 \pm 9,325 \mathrm{Kcal} / 100 \mathrm{~g} M S$ et $466,89 \pm 9,325 \mathrm{Kcal} / 100 \mathrm{~g}$ MS. De même les farines incorporées avec 0 . owariensis sont plus riches en protéines avec $17,69 \pm 3,175 \mathrm{~g} / 100 \mathrm{~g}$ MS pour FMaGO22,5 et $29,73 \pm 5,87 \mathrm{~g} / 100 \mathrm{~g}$ MS pour FMaGO25. La comparaison des valeurs nutritives des farines élaborées à celle du standard montre que la farine FMaGO20 est très proche de la norme FAO/OMS (2008) avec $7,93 \%$ de lipide ; $13,65 \pm 0,347 \%$ de protéine ; 3,55 $\%$ de cendre; $94,68 \pm 2,432 \%$ de Matière sèche; $69,54 \pm 1,054$ de glucide; $404,19 \pm 14,373$ de valeurs variance dimensionnelle afin d'apprécier l'existence de différence statistiquement significative entre les échantillons.

énergétiques. En effet les teneurs en lipides et en protéines des autres farines sont soit trop élevées ou soit trop faibles par rapport à la norme.

Propriétés fonctionnelles des farines élaborées: Les propriétés fonctionnelles des différentes farines étudiées ont été déterminées (Tableau 3). En effet, les caractéristiques fonctionnelles de la farine de maïs sont statistiquement différentes de celles des poudres d'insectes produites. II ressort aussi que la poudre d'O. owariensis présente une capacité d'absorption en eau et en huile plus élevée que celle du $R$. phoenicis respectivement de $176,185 \pm 9,17 \%$ contre $6,667 \pm 1,649 \%$ et $210,728 \pm 1,024 \%$ contre $129,739 \pm 20,639 \%$. Cependant l'activité émulsifiante et la stabilité de l'émulsion de ces deux types d'insectes étudiés sont statistiquement identiques. L'incorporation de poudres d'insectes à un impact positif significatif sur les propriétés fonctionnelles de la farine de maïs germé. La capacité d'absorption en huile (CAH) des farines élaborées augmente. Toutefois, l'analyse statistique effectuée présente des valeurs (CAH) significatives de $136,966 \pm 16,901 \%$ pour FMaGR25; $136,241 \pm 8,432 \%$ pour FMaGRO20 ; 135,892 $\pm 2,777 \%$ pour FMaGO22,5 et $170,958 \pm 19,374 \%$ pour FMaGO25 contre $109,378 \pm$ $9,261 \%$ pour la farine de maïs germé. II en ressort aussi, une baisse de la capacité d'absorption en eau pour toutes les farines formulées. Ainsi la farine FMaGO20 a enregistré la plus faible capacité d'absorption en eau $(91,410 \pm 18,457 \%)$. En revanche, les activités émulsifiantes des farines FMaGR20 $(29,442 \pm 3,847 \%)$, FMaGR25 $(28,114 \pm 1,933 \%)$ et FMaGO25 $(26,989 \pm 0,754 \%)$ augmentent significativement par rapport à celle de la farine de Maïs germé qui est de $24,814 \pm 1,740 \%$. De même cette augmentation significative est observée au niveau de la stabilité de l'émulsion de certaines farines élaborées et passe respectivement de $59,913 \% \pm 7,828 \%$ pour la farine de maïs germé à $69,744 \% \pm 0,444 \%$ pour FMaGR22,5; à $72,692 \pm 8,462$ pour FMaGO20 et à $72,187 \pm 9,703$ pour FMaGO25. 
Tableau 2 : Caractéristiques physico-chimique des farines élaborées

\begin{tabular}{|c|c|c|c|c|c|c|c|c|c|c|c|}
\hline & \multicolumn{3}{|c|}{ Matrices utilisées } & \multicolumn{3}{|c|}{$\begin{array}{l}\text { Farine enrichie avec Rhynchophorus } \\
\text { phoenicis }\end{array}$} & \multicolumn{3}{|c|}{$\begin{array}{l}\text { Farine enrichie avec Oryctes } \\
\text { owariensis }\end{array}$} & \multicolumn{2}{|c|}{$\begin{array}{l}\text { Normes } \\
\text { FAO / OMS }\end{array}$} \\
\hline & MaG & Rhyn & Oryc & $\begin{array}{l}\text { FMaGR } \\
20\end{array}$ & $\begin{array}{l}\text { FMaGR } \\
22.5\end{array}$ & $\begin{array}{l}\text { FMaGR } \\
25\end{array}$ & $\begin{array}{l}\text { FMaGO } \\
20\end{array}$ & $\begin{array}{l}\text { FMaGO } \\
22.5\end{array}$ & $\begin{array}{l}\text { FMaGO } \\
25\end{array}$ & 2006 & 2008 \\
\hline $\mathrm{pH}$ & $\begin{array}{l}4,92 \pm 0,028 \\
a\end{array}$ & $5,83 \pm 0,032 \mathbf{b}$ & $7,41 \pm 0,034 \mathrm{c}$ & $\begin{array}{l}4,84 \pm 0,00 \\
58 \mathrm{a}\end{array}$ & $4,88 \pm 0,032$ & $4,85 \pm 0,035 \mathrm{a}$ & $\begin{array}{l}6,44 \pm 0,049 \\
\text { e }\end{array}$ & $\begin{array}{l}6,58 \pm 0 \\
021 \mathrm{f}\end{array}$ & $\begin{array}{l}6,44 \pm 0,006 \\
\text { e }\end{array}$ & - & - \\
\hline Humidité (\%) & $\begin{array}{l}8,91 \pm 0,992 \\
\text { a }\end{array}$ & $\begin{array}{l}13,03 \pm 2,045 \\
\text { c }\end{array}$ & $9,11 \pm 2,275 \mathbf{a}$ & $\begin{array}{l}7,31 \pm 0,59 \\
8 \mathrm{~b}\end{array}$ & $6 \pm 2,645 b$ & $8 \pm 2,646 a$ & $\begin{array}{l}5,32 \pm 2,432 \\
\text { b }\end{array}$ & $\begin{array}{l}6,38 \pm 1 \\
841 \mathrm{~b}\end{array}$ & $\begin{array}{l}9,69 \pm 3,135 \\
\text { a }\end{array}$ & - & - \\
\hline $\begin{array}{l}\text { Acidité titrable } \\
\text { (meq } / 100 \mathrm{~g} \mathrm{MS} \text { ) }\end{array}$ & $\begin{array}{l}3,733 \pm 0,41 \\
6 \mathrm{a}\end{array}$ & $6,93 \pm 0,923 \mathbf{b}$ & $\begin{array}{l}10,80 \pm 1,424 \\
\text { c }\end{array}$ & $\begin{array}{l}5,73 \pm 0,30 \\
6 \mathbf{b}\end{array}$ & $\begin{array}{l}10,00 \pm 0,00 \\
\text { c }\end{array}$ & $00 \mathrm{c}$ & $10,40 \pm 0,462$ & $\begin{array}{l}11,10 \pm 1 \\
, 052 \mathrm{c}\end{array}$ & $\begin{array}{l}10,00 \pm 1,633 \\
\text { c }\end{array}$ & - & - \\
\hline Lipide (\%) & $\begin{array}{l}4,29 \pm 0,632 \\
\text { a }\end{array}$ & $\begin{array}{l}29,79 \pm 6,589 \\
\text { d }\end{array}$ & $\begin{array}{l}23,31 \pm 1,975 \\
\text { e }\end{array}$ & $\begin{array}{l}15,17 \pm 1,3 \\
32 \text { b }\end{array}$ & $\begin{array}{l}21,24 \pm 0,772 \\
\text { c }\end{array}$ & $19,20 \pm 0,361 \mathrm{c}$ & $7,93 \pm 1,227 \mathbf{f}$ & $\begin{array}{l}13,93 \pm 4 \\
, 739 \mathrm{~b}\end{array}$ & $\begin{array}{l}17,48 \pm 2,195 \\
\text { b }\end{array}$ & 8 & 7 \\
\hline Protéine (\%) & $\begin{array}{l}8,65 \pm 0,192 \\
\text { a }\end{array}$ & $\begin{array}{l}35,64 \pm 0,136 \\
\text { d }\end{array}$ & $\begin{array}{l}47,31 \pm 7,066 \\
\text { e }\end{array}$ & $\begin{array}{l}9,67 \pm 0,14 \\
4 \text { b }\end{array}$ & $\begin{array}{l}10,79 \pm 0,189 \\
\text { b }\end{array}$ & $13,13 \pm 0,095 \mathrm{c}$ & $\begin{array}{l}13,65 \pm 0,347 \\
\text { c }\end{array}$ & $\begin{array}{l}17,69 \pm 3 \\
, 175 \mathrm{f}\end{array}$ & $\begin{array}{l}29,73 \pm 5,87 \\
\text { g }\end{array}$ & 15 & 13 \\
\hline $\begin{array}{l}\text { Vitamine C } \\
\text { (mq/100g MS) }\end{array}$ & $\begin{array}{l}283,07 \pm 17 \\
782 \text { a }\end{array}$ & $\begin{array}{l}415,07 \pm 22,57 \\
9 \mathrm{c}\end{array}$ & $\begin{array}{l}114,84 \pm 10,02 \\
1 \mathrm{~d}\end{array}$ & $\begin{array}{l}385,73 \pm 2 \\
540 \text { b }\end{array}$ & $396 \pm 4,40 b$ & $391,60 \pm 4,40$ b & $\begin{array}{l}119,020 \pm 3,3 \\
21 \mathrm{~d}\end{array}$ & $\begin{array}{l}113,74 \pm \\
4,45 \mathrm{~d}\end{array}$ & $\begin{array}{l}122,54 \pm 6,32 \\
0 \mathrm{~d}\end{array}$ & - & \\
\hline Degré brix (\%) & $\begin{array}{l}5,10 \pm 0,000 \\
\text { a }\end{array}$ & $3,97 \pm 0,058 \mathrm{~d}$ & $\begin{array}{l}8,875 \pm 0,263 \\
\text { e }\end{array}$ & $6 \pm 0,000 \mathrm{~b}$ & $\begin{array}{l}6,50 \pm 0,000 \\
\text { b }\end{array}$ & $5,733 \pm 0,115 \mathrm{c}$ & $\begin{array}{l}3,80 \pm 0,231 \\
\text { d }\end{array}$ & $\begin{array}{l}3,950 \pm 0 \\
, 058 \mathrm{~d}\end{array}$ & $\begin{array}{l}6,45 \pm 0,006 \\
\text { c }\end{array}$ & - & \\
\hline Cendre (\%) & $\begin{array}{l}1,40 \pm 0,20 \\
\text { a }\end{array}$ & $1,46 \pm 0,93 a$ & $\begin{array}{l}10,98 \pm 1,375 \\
\text { c }\end{array}$ & $\begin{array}{l}2,00 \pm 0,86 \\
6 \mathbf{b}\end{array}$ & $\begin{array}{l}1,33 \pm 0,289 \\
\mathbf{a}\end{array}$ & $1,83 \pm 0,286 \mathbf{b}$ & $\begin{array}{l}3,55 \pm 1,144 \\
\text { b }\end{array}$ & $\begin{array}{l}1,75 \pm 0 \\
035 \mathrm{a}\end{array}$ & $\begin{array}{l}3,41 \pm 0,324 \\
\text { b }\end{array}$ & 2.9 & 2 \\
\hline $\begin{array}{l}\text { Matière sèche } \\
(\%)\end{array}$ & $\begin{array}{l}91,09 \pm 0,99 \\
\text { a }\end{array}$ & $\begin{array}{l}86,97 \pm 2,045 \\
\text { b }\end{array}$ & $\begin{array}{l}90,89 \pm 2,275 \\
\text { a }\end{array}$ & $\begin{array}{l}92,69 \pm 0,5 \\
98 \mathrm{a}\end{array}$ & $\begin{array}{l}94,00 \pm 2,645 \\
\text { c }\end{array}$ & $92,00 \pm 2,645 \mathbf{a}$ & $\begin{array}{l}94,68 \pm 2,432 \\
\text { c }\end{array}$ & $\begin{array}{l}93,62 \pm 1 \\
, 841 \mathrm{c}\end{array}$ & $\begin{array}{l}90,31 \pm 3,135 \\
\text { a }\end{array}$ & 95 & 95 \\
\hline Glucide (\%) & $\begin{array}{l}76,74 \pm 1,44 \\
5 a\end{array}$ & $20,08 \pm 8,94 \mathrm{c}$ & $9,32 \pm 8,60$ e & $\begin{array}{l}65,85 \pm 2,0 \\
36 \text { b }\end{array}$ & $\begin{array}{l}62,68 \pm 2,189 \\
\text { b }\end{array}$ & $55,79 \pm 2,742 \mathrm{~d}$ & $\begin{array}{l}69,54 \pm 1,054 \\
\text { a }\end{array}$ & $\begin{array}{l}60,24 \pm 5 \\
, 73 \mathbf{b}\end{array}$ & $\begin{array}{l}42,69 \pm 10,67 \\
7 \mathrm{~d}\end{array}$ & 68 & 68 \\
\hline $\begin{array}{l}\text { VE (Kcal/100g } \\
M S)\end{array}$ & $\begin{array}{l}380,23 \pm 3,6 \\
0 \mathrm{a}\end{array}$ & $\begin{array}{l}490,99 \pm 25,02 \\
7 \mathrm{~d}\end{array}$ & $\begin{array}{l}436,19 \pm 19,16 \\
9 \text { b }\end{array}$ & $\begin{array}{l}438,59 \pm 5, \\
003 \text { b }\end{array}$ & $\begin{array}{l}466,67 \pm 9,32 \\
5 \text { c }\end{array}$ & $\begin{array}{l}466,89 \pm 9,325 \\
\text { c }\end{array}$ & $\begin{array}{l}404,19 \pm 14,3 \\
73 \text { e }\end{array}$ & $\begin{array}{l}442,09 \pm \\
33,908 \\
\text { b }\end{array}$ & $\begin{array}{l}427,532 \pm 15 \\
763 \text { b }\end{array}$ & 400 & 400 \\
\hline
\end{tabular}

Les valeurs avec des lettres alphabétiques différentes sur la même ligne sont statistiquement différentes $(P<0,05)$. MaG : Maïs germé ; Rhyn : Rhynchophorus phoenicis ; Oryc : oryctes owariensis. Farines enrichies au Rhynchophorus phoenicis (FMaGR20; FMaGR22.5 et FMaGR25) farines enrichies à l'Oryctes owariensis (FMaGO20; FMaGO22.5 et FMaG025) 
Angaman et al., J. Appl. Biosci. 2021 Propriétés physico-chimiques, fonctionnelles et microbiologiques de la farine de maïs germé enrichie de larves d'insectes comestibles Rhynchophorus phoenicis et Oryctes owariensis

Tableau 3 : Propriétés fonctionnelles des farines élaborées

\begin{tabular}{|c|c|c|c|c|c|c|c|c|c|}
\hline & MaG & Rhyn & Oryc & FMaGR20 & FMaGR22,5 & FMaGR25 & FMaG020 & FMaG022,5 & FMaGO25 \\
\hline $\mathrm{AE}$ & $\begin{array}{c}24,814 \pm 1,740 \\
\text { a }\end{array}$ & $\begin{array}{l}21,506 \pm 1,92 \\
2 \text { d }\end{array}$ & $\begin{array}{c}21,002 \pm 0,9 \\
55 \mathrm{~d}\end{array}$ & $\begin{array}{c}29,442 \pm 3,847 \\
\text { b }\end{array}$ & $\begin{array}{c}23,487 \pm 1,402 \\
\text { a }\end{array}$ & $\begin{array}{c}28,114 \pm 1,933 \\
\text { c }\end{array}$ & $\begin{array}{c}24,233 \pm 2,055 \\
\mathbf{a}\end{array}$ & $\begin{array}{c}24,188 \pm 4,344 \\
\mathbf{a}\end{array}$ & $\begin{array}{c}26,989 \pm 0,754 \\
\text { c }\end{array}$ \\
\hline $\begin{array}{c}\text { CA } \\
E\end{array}$ & $\begin{array}{c}116,766 \pm \\
0,462 \mathrm{a} \\
\end{array}$ & $\begin{array}{l}6,667 \pm 1,649 \\
\text { c }\end{array}$ & $\begin{array}{c}176,185 \pm 9 \\
17 \mathbf{d}\end{array}$ & $\begin{array}{c}102,314 \pm 3,18 \\
1 \text { b } \\
\end{array}$ & $\begin{array}{c}100,766 \pm 2,50 \\
3 \mathrm{~b} \\
\end{array}$ & $\begin{array}{c}100,320 \pm 1,506 \\
\text { b }\end{array}$ & $\begin{array}{c}91,410 \pm 18,45 \\
7 \mathrm{e}\end{array}$ & $\begin{array}{c}109,448 \pm \\
7,954 \text { b }\end{array}$ & $\begin{array}{c}115,319 \pm 37,10 \\
2 a\end{array}$ \\
\hline $\begin{array}{c}\mathrm{CA} \\
\mathrm{H}\end{array}$ & $\begin{array}{l}109,378 \pm \\
9,261 \mathbf{a}\end{array}$ & $\begin{array}{l}129,739 \pm 20, \\
639 \text { a }\end{array}$ & $\begin{array}{c}210,728 \pm 1 \\
024 \text { c }\end{array}$ & $\begin{array}{l}128,154 \pm 70,9 \\
35 \text { a }\end{array}$ & $\begin{array}{l}112,667 \pm 11,3 \\
72 \text { a }\end{array}$ & $\begin{array}{c}136,966 \pm 16,90 \\
1 \text { b }\end{array}$ & $\begin{array}{c}136,241 \pm 8,43 \\
2 \text { b }\end{array}$ & $\begin{array}{c}135,892 \pm 2,77 \\
7 \text { b }\end{array}$ & $\begin{array}{c}170,958 \pm 19,37 \\
4 \text { b }\end{array}$ \\
\hline SE & $\begin{array}{c}59,913 \pm 7,828 \\
\text { a }\end{array}$ & $\begin{array}{l}81,118 \pm 3,89 \\
3 \mathrm{c}\end{array}$ & $\begin{array}{c}91,477 \pm 6,8 \\
15 \text { c }\end{array}$ & $60,00 \pm 10,00$ a & $\begin{array}{c}69,744 \pm 0,444 \\
\text { b }\end{array}$ & $\begin{array}{c}54,929 \pm 3,390 \\
\text { a }\end{array}$ & $\begin{array}{c}72,692 \pm 8,462 \\
\text { b }\end{array}$ & $\begin{array}{c}60,669 \pm 25,53 \\
0 \mathrm{a}\end{array}$ & $\begin{array}{c}72,187 \pm 9,703 \\
\text { b }\end{array}$ \\
\hline
\end{tabular}

Les valeurs avec des lettres alphabétiques différentes sur la même ligne sont statistiquement différentes ( $<<0,05)$. MaG : Maïs germé ; Rhyn : Rhynchophorus phoenicis ; Oryc : Oryctes owariensis. Farines enrichies avec Rhynchophorus phoenicis (FMaGR20; FMaGR22,5 et FMaGR25) farines enrichies avec Oryctes owariensis (FMaGO20;

FMaG022,5 et FMaGO25). 
Angaman et al., J. Appl. Biosci. 2021 Propriétés physico-chimiques, fonctionnelles et microbiologiques de la farine de maïs germé enrichie de larves d'insectes comestibles Rhynchophorus phoenicis et Oryctes owariensis

Qualité microbiologique des farines élaborées : Le Tableau 4 présente la qualité microbiologique des différentes farines de maïs enrichies aux insectes. Une charge fongique de $3,465.10^{3} \mathrm{UFC} / \mathrm{g}$ a été détectée seulement chez la farine FMaGR25. De plus, les coliformes totaux ont été dénombrés uniquement dans les farines Oryc ; FMaGO22.5 et FMaGO25 avec des charges respectives de 3,5.101 UFC/g ; 2,53.101 UFC/g et $1,955.10^{2} \mathrm{UFC} / \mathrm{g}$. Cependant, Les charges des champignons et des coliformes totaux détectées sont inférieures aux normes microbiologiques applicables aux farines infantiles (Codex Stan 74, 1981). Aussi, Les entérobactéries et les salmonelles n'ont pas été détectées.

Tableau 4 : Qualité microbiologique des farines.

\begin{tabular}{|l|c|c|c|c|}
\hline Farines & Moisissures et Levures & Coliformes totaux & Entérobactéries & Salmonella \\
\hline MaG & $<1$ & $<1$ & $<1$ & Absence \\
\hline Rhyn & $<1$ & $<1$ & $<1$ & Absence \\
\hline Oryc & $<1$ & $3,5.10^{1}$ & $<1$ & Absence \\
\hline FMaGR20 & $<1$ & $<1$ & $<1$ & Absence \\
\hline FMaGR22,5 & $<1$ & $<$ & $<1$ & Absence \\
\hline FMaGR25 & $3,465.10^{3}$ & $<$ & $<1$ & Absence \\
\hline FMaGO20 & $<1$ & $<$ & $<1$ & Absence \\
\hline FMaGO22,5 & $<1$ & $2,53.10^{1}$ & $<1$ & Absence \\
\hline FMaGO25 & $<1$ & $1,955.10^{2}$ & $<10^{3}$ & Absence \\
\hline $\begin{array}{l}\text { Norme } \\
\text { Microbiologique }\end{array}$ & $<10^{5}$ & $<10^{3}$ & & Absence \\
\hline
\end{tabular}

MaG : Maïs germé ; Rhyn : Rhynchophorus phoenicis ; Oryc: Oryctes owariensis. Farines enrichies avec Rhynchophorus phoenicis (FMaGR20 ; FMaGR22,5 et FMaGR25) farines enrichies avec Oryctes owariensis (FMaGO20 ; FMaGO22,5 et FMaG025).

\section{DISCUSSION}

L'incorporation des poudres de larves de $R$. phoenicis ou de 0 . owariensis dans la farine de maïs a eu une influence sur les propriétés physico-chimiques. L'augmentation de l'acidité des farines de maïs enrichie a été également observée par Niaba et al. (2014). Selon Soro et al. (2013) une augmentation de l'acidité pourrait faciliter la conservation des farines produites. Le faible taux d'humidité enregistré pourrait être dû au séchage préalable des matrices utilisées. FAO/OMS (2006), préconise un niveau d'humidité inférieur à $10 \%$ pour conserver les produits farineux à des durées raisonnables. L'ajout de la poudre d'insecte a favorisé une augmentation de la teneur en cendre variant de $(1,4$ à $3,55 \%$ ). Cette augmentation est aussi rapportée par Ajayi et al. (2012) ; Shiriki et al. (2015) qui indiquent un enrichissement de ces farines en minéraux. En effet la teneur en cendre est une estimation approximative de la teneur en minéraux d'un produit (Adegunwa et al., 2014). En ce qui concerne la valeur nutritive telle que les protéines, lipides et valeur énergétique, la teneur augmente significativement avec le taux d'incorporation des insectes. Cette constatation est corroborée par les études de Banjo et al. (2006) ; Kinyuru et al. (2009); Niaba et al. (2014), lors de la formulation de farines alimentaires. Selon ces auteurs cette variation significative en éléments nutritifs pourrait être attribuée aux différentes proportions de poudre de larves d'insectes incorporée lors de la formulation de farines enrichies. Les larves d'insectes comestibles sont de véritables sources d'éléments nutritifs capables de compenser le déséquilibre alimentaire. La teneur en protéine des farines enrichies varie de $9,67 \pm 0,14$ à $29,73 \pm 5,87 \%$. Cette teneur est supérieure à celle obtenue par Sika et al. (2019) (10,15 à 14,51\%) lors de l'enrichissement du maïs au Safou. Aussi, les teneurs en protéines les plus élevées obtenues pour les farines FMaGO20; FMaGO22,5 et FMaGO25 enrichies avec 0. owariensis pourraient être dues au fait que ces larves ont des proportions plus élevées en protéines que celle du $R$. phoenicis. En générale, les protéines d'insectes sont connues pour être d'une bonne digestibilité contenant certains acides aminés essentiels en quantité appréciable (Food and Nutrition Board, 1980). Ekpo \& Onigbinde, (2005) ont signalé des taux élevés de leucine, 
de lysine et de thréonine chez les larves d'insectes. La consommation de ces farines pourrait faciliter la croissance des enfants. Selon Soro et al. (2013), un aliment riche en protéine facilite la séparation des tissus et la musculation. Ces aliments sont donc importants pendant la croissance et la grossesse. De plus, le RDA (Recommended Dietery Allowance) recommande que les nourrissons (0 à 3 ans) consomment environ $16 \mathrm{~g}$ de protéine par jour. Ainsi, un repas de $100 \mathrm{~g}$ constitué de farine de maïs enrichie aux larves de 0 . owariensis pourrait donc réponde à leur besoin en protéine. La teneur en protéine des farines FMaGO22,5 $(17,69 \%)$ et FMaGO25 $(29,73 \%)$ est supérieure à celle de la farine infantile commerciale BLEDINE (16,69\%) (Soro et al., 2013). Contrairement aux protéines, les larves du $R$. phoenicis présentent une teneur plus élevée en lipides par rapport à $\mathrm{O}$. Owariensis. Ceci pourrait donc expliquer les teneurs les plus élevées en lipides pour les farines FMaGR20 (15,17 $\pm 1,33 \%)$; FMaGR22,5 (21,24 $\pm 0,77 \%)$ et FMaGR25 $(19,20 \pm 0,36 \%)$. Les farines de maïs enrichies avec $R$. phoenicis pourraient présenter une meilleure appétence sur le plan sensoriel. En effet, la matière grasse absorbe et conserve leur arôme (Aiyesanmi et al., 1996). Toutefois, en dehors du FMaGO20, l'ensemble des farines élaborées est conforme aux normes (10 à 25\%) de lipides établis par le Codex Alimentarius (CAC/GL08-1991). Selon Fasasi (2009), une faible teneur en matière grasse dans un produit sec contribuerait à augmenter la durée de conservation de l'échantillon en diminuant les risques de rancissement. Tandis qu'un produit à haute teneur en matière grasse aurait une valeur énergétique plus élevée et favoriserait l'oxydation. Ceci pourrait expliquer les valeurs énergétiques plus élevées pour les farines FMAGR22,5 (466,6 $\pm 9,32 \mathrm{Kcal} / 100 \mathrm{gMS})$ et FMaGR25 $(466,86 \pm 9,32)$. De plus, les valeurs énergétiques obtenues dans cette étude sont plus élevées que celles de Sika et al. (2019) pour l'enrichissement des maïs au safou (391,94 à 400,86 Kcal/100gMS). Aussi, ces valeurs sont-elles supérieures aux recommandations de l'OMS pour l'aliments de sevrage ( $400 \mathrm{Kcal} / 100 \mathrm{~g} \mathrm{MS}$ ) (Lutter et al., 2003). Cependant, une baisse de la teneur en glucide est observée. Cette baisse consécutive pourrait être due à la faible teneur en sucre des larves de $R$. phoenicis et O. owariensis (Niaba et al., 2014 ; Koffi et al., 2017). De plus, un régime à base d'insectes peut réduire l'apport calorique entrainant une perte de poids souhaité (Kappagoda et al., 2004). Des recherches récentes ont révélé que les insectes contiendraient des quantités considérables de polysaccharides qui pourraient améliorer la fonction immunitaire du corps humain (Long et al., 2007). En dehors de la FMaGR25 et FMaGO20; les farines élaborées présentent des teneurs proches de la norme de $68 \%$ établies par le Codex Alimentarus (CAC/G208-1991). De façon générale, les insectes comestibles amélioreraient la qualité nutritionnelle de la farine de maïs. Ces résultats sont corroborés par Assielou et al. (2015) ; Niaba et al. (2014). Cependant seule la farine (FMaGO20) a une composition biochimique la plus proches du standard (FAO/OMS2006/2008). Les propriétés fonctionnelles sont très importantes dans les formulations alimentaires (Oyarekua \& Adeyere, 2008). La capacité d'absorption en eau $(210,72 \pm 1,02)$ et la capacité d'absorption en huile $(176,18 \pm 9,17)$ de 0 . owariensis est supérieure à celle du $R$. phoenicis. Cela peut être dû à la présence de constituants hydrophiles. En effet, les farines à haute capacité d'absorption d'eau ont plus de constituants hydrophiles comme les polysaccharides. Aussi, une faible teneur en humidité de ces larves séchées a également amélioré leur CAE (Aremu et al., 2009). Assielou et al. (2015) et Koffi et al. (2017) ont obtenu une CAE plus élevée pour l'espèces 0 . owariensis $(220,33 \%)$ et $R$. phoenicis $(281,73 \%)$. Selon Assielou et al. (2015), la capacité d'absorption en huile élevée est due à la présence d'acides aminés apolaires dans les farines de 0 . owariensis. Cette $\mathrm{CAH}$ indique que la vitesse à laquelle la protéine se lie aux graisses dans la formulation des aliments. La capacité d'absorption en huile du $R$. phoenicis et celle de 0 . owariensis est inférieure à celle obtenue par Koffi et al. (2017) respectivement 139\%; $265,90 \%$. Toutefois, elle reste très utile dans la formulation des aliments tels que les saucisses et produits boulangers (Adebowale \& Lawal, 2004). L'incorporation de la poudre d'insecte impacte significativement l'ensemble des propriétés fonctionnelles de la farine de maïs. Selon Nelson-Quartey et al. (2007), la présence des lipides en grande quantité dans la farine réduit la capacité de liaison de l'eau à des substances particulières limitant ainsi la CAE. Raison qui explique la diminution progressive de la capacité d'absorption en eau des différentes formulations, vue leur enrichissement en lipide. D'autre part, Oti \& Akobundu (2008) rapportent que, plus le niveau de protéine dénaturé est bas, plus il faut le temps pour se mouiller ou s'imprégner d'eau. La capacité d'absorption en eau des farines formulées $(91,41 \%$ à $109,44 \%)$ est inférieure à celles des farines d'igname Niébé (128\%) et de pois chiche (136\%) obtenu par Ghavidel et Prakash (2006), Kaur \& Singh (2005). La capacité d'absorption en huile augmente avec l'incorporation de la poudre des larves d'insectes comestibles passant de $109,38 \%$ pour le Maïs 
germé pour un maximum de $170,95 \%$ pour la FMGO25. Cette $\mathrm{CAH}$, est supérieure à celle de Ojinnaka et al. (2016) lors de l'enrichissement du Maïs au R. phoenicis (104 à 139\%). La capacité d'absorption en huile est importante dans la conception des aliments effectivement la matière grasse agit comme un agent de conservation de la saveur et augmente la palatabilité des aliments (Aremu et al., 2009). Pour Adebowale \& Lawal (2004) les variations de la présence des chaînes latérales non polaires, qui pourraient lier les chaînes latérales d'hydrocarbures du pétrole parmi les farines expliqueraient les différences de capacité de liaison d'huile des farines. Ainsi, les farines formulées pourraient être de bon conservateur de saveur et d'arôme lors de la fabrication de produit alimentaire. Une augmentation de l'activité émulsifiante et de la stabilité de l'émulsion est aussi observée lors de l'incorporation des larves d'insectes surtout au niveau des farines enrichies avec 0 .

\section{CONCLUSION ET APPLICATION DES RÉSULTATS}

Cette étude a permis de montrer que les larves de Rhynchophorus phoenicis et Oryctes owariensis sont de véritables sources d'éléments nutritifs. Cette source nutritionnelle est constituée de protéines $(35,64 \%$ à $4731 \%)$; de matière grasses $(23,31 \%$ à $29,79 \%)$, de cendre $(1,46 \%$ à $10,98 \%)$ d'où leur richesse en minéraux et leur bonne valeur énergétique allant jusqu'à 490,99 $\mathrm{Kcal} / 100 \mathrm{~g} \mathrm{MS}$. Leur incorporation à différent proportion $(20 \% ; 22,5 \%$ et $25 \%)$ a permis d'élaborer des farines de maïs germé enrichies aux insectes comestibles avec une valeur énergétique variant de 368,23 à 466,89 $\mathrm{Kcal} / 100 \mathrm{~g} \mathrm{MS}$. En dehors de la qualité nutritionnelle ces

\section{REFERENCES}

AACC, 1984. Damaged starch method 76-30A. In: Approved methods of the American Ass of Cereal Chemists. $8^{\text {th }}$ ed. St. Paul, MN, p. 2.

Adebowale KO, Lawal OS, 2004. Comparative study of the functional properties of bambara groundnut, jack bean and mucuna bean flours. Food Research International 37 : 355-364.

Adegunwa MO, Ganiyu AA, Bakare HA., Adebowale, A. A. (2014). Quality evaluation of composite millet-wheat Chin-chin. Agriculture Biology Journal of North America, 5(1) : 33-39.

Aiyesanmi AF, Oguntokun MO, 1996. Nutrient composition of Dioclea reflex a seed - an underutilized edible legume. La Rivista Italiana Delle Sostanze Grasse, 73 : 521-523.

Ajayi OE, 2012. Biochemical analyses and nutritional content of flour castes of subterranean termites, owariensis. Cette augmentation est probablement dû aux propriétés des protéines à conserver la mousse. Ceci peut donc s'avérer bénéfique pour la consistance et l'appétence des aliments (Akubor et al., 2000). L'évaluation de la qualité microbiologique des farines élaborées a présenté des résultats satisfaisants. En effet, aucune colonie de Salmonella n'a été observée. De plus, les charges des moisissures et des levures, ainsi que celles des coliformes totaux enregistrés dans certaines farines étaient inférieures à la norme microbiologique respectivement de $10^{5} \mathrm{UFC} / g$ et de $10^{3} \mathrm{UFC} / g$ pour les farines du codex Stan (74-1981). Cette faible charge pourrait s'expliquer par le faible taux d'humidité dû à un séchage préalable des matrices utilisées $(R$. phoenicis, O. owariensis et maïs germé) (Niaba et al., 2014 ; Sika et al., 2019). Ainsi, Ces résultats pourraient traduire le respect des bonnes pratiques d'hygiène au cours de la fabrication des farines.

farines ont présenté une qualité fonctionnelle et microbiologique satisfaisante pouvant permettre la formulation d'aliment de bonne qualité organoleptique. Ainsi, ces formulations pourraient être des composants du régime alimentaire de populations pour lesquelles les régimes alimentaires seraient constamment déficients en protéines et en matière grasse. Toutefois seule la farine $\mathrm{FMaGO} 20$ présente des valeurs proches de celle de la farine standard (FAO/OMS 2008) pour les farines infantiles. De ce fait, elle pourrait être recommandée pour les nourrissons afin de réduire des maladies liées aux carences nutritives.

Macrotermes subhyalims (Rarnbur) (Isoptera: termitidae) : differences in digestibility and anti nutrient contents among castes. International journal of biology 4 (4) : 54-59.

Akubor PI, Isolokwu PC, Ugbane O, Onimawo IA, 2000. Proximate composition and functional properties of African breadfruit kemel and wheat flour blends. Food Research International 33 : 707-712.

AOAC, 1990. Official methods of Analysis of the Association of Analytical Chemists (15 th Edition) AOAC. Washington, DC, USA, $648 \mathrm{p}$.

AOAC, 2000. Official Methods of Analysis of the Association of Analytical Chemists (17th Edition) AOAC Washington, DC, USA.

Aremu MO, Basu SK, Gyar SD, Goyal A., Bhowmik PK., Datta BS, 2009. Proximate composition and 
functional properties of mushroom flours from Ganoderma spp., Omphalotus olearius (DC.) Sing. and Hebeloma mesophaeum (Pers.) Quél. used in Nasarawa state, Nigeria. Malaysian Journal of Nutrition, 15(2) : 233-241.

Assiélou B, Due EA, Koffi MD, Dabonné S, Kouamé PL, 2015. Oryctes owariensis Larvae as good alternative protein source : nutritional and functional properties. Annual Research and Review in Biology, 8(3) : 1-9.

Atwater WO, Benedict FA, 1902. Experiments on the metabolism of Matter and Energy in the Human Body.

Banjo AD, Lawal OA, Songonuga EA, 2006.The nutritional value of fourteen species of edible insects in southwestern Nigeria. African Journal of Biotechnology 5 : 298-301.

BIPEA, 1976. Recueil des méthodes d'analyse des communautés européennes. Bureau Interprofessionnel d'Études Analytiques, Gennevilliers. France.

CODEX ALIMENTARIUS, 1991. Organisation des Nations unies pour la santé. Rome.

Codex Alimentarius, 1981. Standard for processed cereal based from infant and young student

EDS-MICS, 2012. MEASURE DHS, ICF International Calverton, Maryland, USA

Ehounou G, Ouali-N'goran S, Niassy S, 2018. Evaluation de l'entomophagie à Abidjan (Côte d'Ivoire, Afrique de l'Ouest). African Journal Food Science. 12(1) : 6-14.

Elgamouz S, 2016. Monitoring the content of vitamin C in an industrial juice. Master thesis, Sciences and Techniques: CMBA chemistry of bioactive molecules, from Sidi Mohammed Ben Abdellah University, Morocco ;

FAO, 2019. Rapport sur l'état de la sécurité alimentaire et de la nutrition dans le monde.

FAO, FIDA, OMS, PAM, UNICEF, 2019. The State of Food Security and Nutrition in the World. Guard against economic slowdowns and downturns. Rome.

FAO/OMS, 2006. Programme mixte FAO/OMS sur les normes alimentaires. Rapport de la vingtseptième session du comité du codex sur la nutrition et les aliments diététiques ou de régime. FAO/OMS. ALINORM 06/29/26. 105p.

Fasasi OS, 2009. Proximate, Antinutritional factors and functional properties of processed pear millet. Journal of Food Technology, $7:$ 92-97.
Food and Nutrition Board, 1980. Recommended Dietary Allowances 9th ed. National Academy of Sciences/National Research Council, National Academy Press, Washington D.C.

Ghavidel RA, Prakash J, 2006. Effect of germination and dehulling on functional properties of 1egumes fleurs. Journal of Science of Food and Agriculture, 86 : 1189-1195.

Idolo I, 2010. Nutritional and Quality Attributes of Wheat Buns Enriched with the Larvae of Rhynchophorus phoenicis F. Pakistan Journal of Nutrition, 9(11) : 1043-1046.

INS et ICF, 2012. Enquête Démographique Et De Santé Et à Indicateurs Multiples De Côte d'Ivoire 2011-2012. Calverton, Maryland, USA : Institut National de la Statistique et ICF International.

ISO 4832, 2006. Microbiologie des aliments, méthode horizontale pour le dénombrement des coliformes. Méthode par comptage des colonies. International Standardization Organization.

ISO 4833, 2003. Microbiologie des aliments. Méthode horizontale pour le dénombrement des microorganismes; technique de comptage des colonies à $30^{\circ} \mathrm{C}$. International Standardization Organization.

ISO 7937, 2004. Microbiologie des aliments. Méthode horizontale pour le dénombrement de Clostridium perfringens ; Technique par comptage des colonies. International Standardization Organization.

Kappagoda TC, Hyson AD, Amsterdam AE, 2004. Lowcarbohydrate-high-protein diets is there a place for them in clinical cardiology?. Journal of the American College of Cardiology 43(5) :725-730.

Kaur M, Singh N, 2005. Studies on functional, thermal and pasting properties of flours from different chickpea (Cicer arietinum L.) cultivars. Journal of Food Chemistry, 91 : 403-411

Kinyuru JN, Kenji GM, Njoroge MS, 2009. Process development, nutrition and sensory qualities of wheat buns enriched with edible termites (Macrotermes subhylanus) from Lake Victoria region, Kenya. African Journal of Food Agriculture Nutrition and Development, 9(8): 1739-1750.

Koffi DM, Cissé M, Koua GA, Niamke SL, 2017. Nutritional and functional properties of flour from the palm (Elaeis guineensis) weevil Rhynchophorus phoenicis larvae consumed as 
protein source in south Côte d'lvoire. The Annals of the University Dunarea de Jos of Galati Fascicle VI- Food Technology, 41(1), 919

Kone S, Soro D, Koffi KE, 2019. Formulation and physicochemical characterization of infant flour compound: Attiéké - Cashew kernel flour. International Journal of Innovation and Applied Studies, 25 : 700-708.

Le Gall P, 2015. Les insectes comestibles : des pratiques ancestrales à un enjeu d'avenir pour la sécurité alimentaire et l'économie en Afrique Subsaharienne. AAIS 21ème Conférence de Association Africaine des Entomologistes, 1923 octobre 2015 Cotonou, Bénin.

Lin MJY, Humbert ES, Sosulski FW, 1974. Certain functional properties of sunflower meal products. Journal of Food Science, 39 : 368370.

Long S, Ying F, Zhao H, Tao M, Xin Z, 2007. Studies on alkaline solution extraction of polysaccharide from silkworm pupa and its immunomodulating activities. Forest Research, 20(6) : 782-786.

Lutter CK, Dewey KG, 2003. Proposed Nutrient Composition for Fortified Complementary Foods. Journal of Nutrition, 133 : 3011-3020.

Monrose GS, 2009. Standardization of a Chadeque Jam Formulation and Assessment of Physicochemical, Microbiological and Sensory Parameters. State University of Haiti (UEH / FAMV) Agricultural Engineer.

Nelson-Quartey FC, Amagloh FK, Oduro IN, Ellis WO, 2007. Formulation of an infant food based on breadfruit (Artocarpus altilis) and breadnut (Artocarpus camansi). Acta horticulturae 757 :215-224.

NF V08-059, 2002. Microbiologie des aliments. Dénombrement des levures et moisissures par comptage des colonies à $25^{\circ} \mathrm{C}$. Méthode de routine.

NF V08-060, 2009. Microbiologie des aliments. Dénombrement des coliformes thermotolérants par comptage des colonies obtenues à $44^{\circ} \mathrm{C}$.

NIABA KPV, 2014. Potentialités alimentaires et nutritionnelles de Macrotermes subhyalinus ailé en côte d'ivoire THESE Pour l'obtention du grade de Docteur en Sciences et Technologie des Aliments de l'Université Nangui Abrogoua $149 p$

Ojinnaka MC, Odimegwu EN, llechukwu R, 2016. Functional properties of flour and starch from two cultivars of aerial yam (Dioscorea bulbifera) in South East Nigeria. Journal of Agriculture and Veterinary Science, 9(8) :22-25.

Oti E, Akobundu ENT, 2008. Potentials of cocoyamsoybean-crayfish mixtures in complementary feeding. Nigerian. Agricultural Journal, 39 : 137-145.

Oyarekua MA, Adeyeye El, 2008. Comparative evaluation of the nutritional quality, functional properties and amino acid profile of cofermented maize/cowpea and sorghun/cowpea Ogi as infant complementary food. Asian Journal of Clinical Nutrition, 1: 31- 39.

Shiriki D, Igyor MA, Gernah DI, 2015. Nutritional evaluation of complementary food formulations from maize, soybean and peanut fortified with Moringa oleifera leaf powder. Food and Nutrition Sciences, 6: 494-500.

Sika AE, Kadji BRL, Dje M, Kone FTM, Dabonne S, Koffl-Nevry AR, 2019. Qualité nutritionnelle, microbiologique et organoleptique de farines composées à base de maïs (Zea mays) et de safou (Dacryodes edulis) produites en Côte d'Ivoire. International Journal Biological and Chemical Sciences, 13(1) : 325-337.

Soro S, Konan G, Elleingand E, N'guessan D, Koffi E, 2013. Formulation d'aliments infantiles à base de farines d'igname enrichies au soja. African Journal of Food Agriculture Nutrition and Development, 3(5) : 8313-8339.

Sosulski FW, 1962. La méthode de centrifugation pour la détermination de l'absorption de la farine de blé de force roux de printemps. Cereal Chemistry, $39: 344-350$. 